\title{
Front line treatment of elderly multiple myeloma in the era of novel agents
}

\author{
Marie-Dominique Venon ${ }^{2}$ \\ Aldo M Roccaro ${ }^{1,3}$ \\ Julie Gay ${ }^{2}$ \\ Anne-Sophie Moreau ${ }^{1,2}$ \\ Remy Dulery ${ }^{2}$ \\ Thierry Facon ${ }^{2}$ \\ Irene M Ghobrial' \\ Xavier Leleu ${ }^{1,2}$ \\ 'Department of Medical Oncology, \\ Dana-Farber Cancer Institute \\ and Harvard Medical School, Boston, \\ MA, USA; ${ }^{2}$ Service des Maladies du \\ Sang, Hopital Huriez, CHRU, Lille, \\ France; ${ }^{3}$ Units of Blood Diseases and \\ Cell Therapies, University of Bresica, \\ Medical School, Bresica, Italy
}

\begin{abstract}
Melphalan combined with prednisone (MP) has long been the historical treatment of reference for a large proportion of elderly myeloma (MM) patients ineligible for autologous stem cell transplantation, and is still the backbone of new regimens that include the new era of novel agents. Melphalan-prednisone-thalidomide (MPT) and melphalan-prednisone-bortezomib (Velcade ${ }^{\circledR}$, MPV), proved superior to MP, currently appear to be the treatments of choice for this population. In the near future melphalan-prednisone-lenalidomide (Revlimid ${ }^{\circledR}, M P R$ ) will also provide a third therapeutic option (MPT, MPV, and MPR), in elderly multiple myeloma, eventually. These options could lead to more personalized treatment approaches, based on patient comorbidities, as the three novel agents have somewhat different toxicity profiles. Dexamethasone-based regimen is another option and questions regarding the relative efficacy of melphalan-based versus low-dose dexamethasone-based regimens will require randomized phase III trials. More intensive approaches with new drug combinations or with the incorporation of polyethylene glycolated (PEGylated) liposomal doxorubicin will also require additional studies. Additionally, the important issue of maintenance treatment needs to be further investigated. These new and emerging therapies offer multiple effective treatment options for MM patients and greatly enhanced treatment strategies for clinicians.
\end{abstract}

Keywords: multiple myeloma, elderly, bortezomib, thalidomide, revlimid, IMiDs, supportive care

\section{Introduction}

Multiple myeloma (MM) is the second most common hematologic malignancy and a disease of the elderly. ${ }^{1}$ The annual age-specific incidence of MM increased considerably with age, up to $>40 / 100,000$ for persons $>80$ years of age in some series. The median age at diagnosis for multiple myeloma is 71 and around $65 \%$ of all new cases of MM will be older than 65 years. The number of older patients with this disease is expected to rise over time as a consequence of the increased life expectancy of the normal population. ${ }^{2}$ Myeloma is the cause of death in $75 \%$ of patients older than 65 years with MM.

The recent report from the Surveillance, Epidemiology, and End Results (SEER) Program in patients with myeloma pointed out the gap between young and elderly patients. ${ }^{3}$ From 1990-1992 to 2002-2004, a major improvement of long-term survival had been achieved in younger patients. In contrast, no improvement in survival was observed in patients over 70 years of age and only a modest improvement in patients between 60 and 69 years of age.

Diverse factors including comorbidity, lower performance status, decreased physiologic reserve, and potential undertreatment contribute to this poor outcome, although supportive care of these patients has improved in recent years. ${ }^{4,5}$ However, the concerted action of specific myeloma therapeutics, including most recent progresses in novel agents, and supportive therapies can significantly improve the quality of life 
of myeloma patients. ${ }^{6}$ At the time of diagnosis, patients with MM can be divided into those who either are asymptomatic (approximately $25 \%$ of patients) or symptomatic, these latter requiring a prompt chemotherapy-based intervention. High-dose melphalan with autologous stem cell transplantation (ASCT) is considered the standard of care for patients younger than 65 years of age. Recent studies have suggested that ASCT is safe in some patients over the age of 65 , including some rare patients over the age of 70 , but it does not represent routine practice. ${ }^{7-9}$ Combination chemotherapy with melphalan and prednisone (MP) has been used since the 1960s and, as recently as two years ago, remained the most widely accepted treatment option for elderly patients ineligible for high-dose therapy. ${ }^{10-12}$ More complex combinations with alkylating agents have been substituted but often with added toxicity and inconvenience and no survival advantage over MP. ${ }^{13}$ Dexamethasone (Dex)-based regimens have provided other options. ${ }^{12,14,15}$ Overall, two different backbones have been used for the development of new combinations; MP, mainly in Europe, and Dex, more frequently in North America.

\section{Melphalan-prednisone-based combinations $\mathrm{MP}+$ thalidomide}

In the wake of the activity of thalidomide noted in the late 1990s against relapsed/refractory myeloma, ${ }^{16}$ the drug was then rapidly moved up to the first line, in combination with MP. Following promising results achieved in a phase II study, ${ }^{17}$ the MPT combination was evaluated against MP in several phase III randomized studies as a front-line therapeutic in elderly myeloma patients. The results of two studies, one from the GIMEMA (Groupe Italiano Malattie Ematologiche dell'Adulto, 255 patients) and one from the Intergroupe Francophone du Myélome (IFM; IFM 99-06, 447 patients) are now available. ${ }^{18-20}$ Two additional trials have recently reported results from phase III placebo-controlled studies comparing MPT with MP-placebo: one from the IFM (IFM 01-01, 232 patients) and the other from the Nordic Myeloma Study Group (NMSG; 362 patients). ${ }^{21,22}$ Other studies are ongoing from the HOVON and GIMEMA study groups. $^{23,24}$

\section{Trial designs}

In the IFM99-06 trial, all patients were between 65 and 75 years of age. ${ }^{19}$ The MP regimen consisted of twelve 6-week cycles with melphalan $0.25 \mathrm{mg} / \mathrm{kg}$ and prednisone $2 \mathrm{mg} / \mathrm{kg} /$ day, days $1-4$. In the MPT arm, the same MP regimen was delivered plus thalidomide at the maximum tolerated dose not exceeding $400 \mathrm{mg}$ daily. The thalidomide was stopped at the end of MP (no maintenance phase). The IFM 99-06 trial also incorporated a third arm with standard induction : 2 cycles of vincristine-doxorubicine-dexamethasone (VAD), cyclophosphamide $3 \mathrm{~g} / \mathrm{m}^{2}$ and stem cell mobilization and intermediate dose melphalan $100 \mathrm{mg} / \mathrm{m}^{2}$ (MEL100) with stem cell transplantation (2 consecutive cycles of MEL100). The study was therefore the first and only study to evaluate thalidomide in combination with standard MP against both standard MP alone and stem cell transplantation with a reduced intensity conditioning regimen.

In the GIMEMA trial comparing MP with MPT, patients were between 60 and 85 years of age. ${ }^{18,25}$ The MP regimen consisted of six 4-week cycles with melphalan $4 \mathrm{mg} / \mathrm{m}^{2}$ days 1-7. The MPT regimen consisted of the same MP regimen plus thalidomide $100 \mathrm{mg}$ daily until progression. The primary endpoint was response rate and event-free survival.

The IFM 01-01 double-blind trial, comparing MP plus placebo with MPT, was remarkable in that it was exclusively devoted to patients over the age of 75 (median age 78.5 years, one third of patients over 80). ${ }^{21}$ Even though these patients represent more than $20 \%$ of MM patients, there are rarely considered for enrolment in clinical trials. As in the IFM 99-06, twelve 6-week cycles of MP were given but with a lower dose of melphalan $(0.2 \mathrm{mg} / \mathrm{kg}$ days $1-4)$. Thalidomide was given at $100 \mathrm{mg}$ daily until the end of MP (no maintenance).

In the NMSG trial, mean age was 75 years (49-92) the MP regimen consisted of 6-week cycles until plateau phase with melphalan $0.25 \mathrm{mg} / \mathrm{kg}$. Thalidomide was started at $200 \mathrm{mg}$ daily and escalated to $400 \mathrm{mg}$ daily. Patients also received thalidomide maintenance treatment in plateau phase at $200 \mathrm{mg}$ daily. ${ }^{22}$ In this study, as well as in IFM studies, the primary endpoint was overall survival.

\section{Study results}

In the first two published phase III studies (GIMEMA and IFM 99-06), the superiority of MPT over MP was clearly demonstrated on the basis of response, including complete response (CR), and progression-free survival (Table 1). ${ }^{18-20}$ MPT response results were concordant with $13 \%$ and $15.5 \%$ CR rates in the GIMEMA and IFM 99-06 studies, respectively, and an identical $76 \%$ overall response rate. In the IFM 01-01 study, response results of MPT arm were slightly inferior but still significantly superior to those of MP, with a $61 \%$ response rate and a $7 \% \mathrm{CR}$ rate. ${ }^{21}$ 
Table I Comparative response and survival across trials of MPT compared with MP

\begin{tabular}{|c|c|c|c|c|c|c|}
\hline Trial & Regimen & $\mathbf{N}$ & CR (\%) & $\geq$ PR (\%) & PFS (mo) & OS (mo) \\
\hline \multirow[t]{3}{*}{ IFM 99-06'19 } & MPT & 125 & 13 & 76 & 27.5 & 51.6 \\
\hline & MP & 196 & 2 & 35 & 17.8 & 33.2 \\
\hline & MEL 100 & 126 & 18 & 65 & 19.4 & 38.3 \\
\hline \multirow[t]{2}{*}{ GIMEMA $^{20}$} & MPT & 129 & 15.6 & 76 & 21.8 & 45 \\
\hline & MP & 126 & 3.7 & 48 & 15.5 & 47.6 \\
\hline \multirow[t]{2}{*}{ IFM 0I-0I } & MPT & 113 & 7 & 61 & 24.1 & 45.3 \\
\hline & MP & 116 & 1 & 31 & 19.0 & 27.7 \\
\hline \multirow[t]{2}{*}{ NMSG Study 22} & MPT & 182 & 13 & 57 & 16 & 29 \\
\hline & MP & 175 & 4 & 40 & 14 & 33 \\
\hline \multirow[t]{2}{*}{ HOVON Study $* 23$} & MPT & 152 & 1 & 63 & - & - \\
\hline & MP & 149 & 1 & 47 & - & - \\
\hline
\end{tabular}

Notes: For PFS and OS, results are presented in median (months). *Results from interim analysis. MELI00: High-dose melphalan I00 mg/m².

Abbreviations: $\mathrm{N}$, number of patients; $\mathrm{CR}$, complete response; PR, partial response; PFS, progression-free survival; OS, overall survival.

Median progression-free survival times with MPT were similar in all three studies, ranging from 24 to 27.5 months. Concerning overall survival, only a nonsignificant survival advantage was noted with MP arm in the GIMEMA study. In both IFM studies, the progression-free survival advantage observed with MPT translated into a significant overall survival advantage. In the Nordic Study, the addition of thalidomide to MP resulted in a significant advantage in terms of response rate and time to progression compared with MP. ${ }^{22}$ However, these favorable results did not translate into an overall survival advantage. The study was hampered by use of higher doses of melphalan and thalidomide in a population with a high proportion of patients over 75 years of age (mean age 74.5 years) and approximately one third of patients with poor performance status (World Health Organization [WHO] performance status of 3 or 4 in $30 \%$ of patients). These characteristics likely contributed to more frequent early deaths in the MPT group, especially in the oldest patients. Of note effective median or mean durations of thalidomide treatment have been somewhat different across trials; 8, 11, and 13.5 months (median) in GIMEMA, IFM 99-06, and IFM 01-01, respectively, and only 6 months (mean) in the NMSG Study.

In the IFM 99-06 study, MPT was also found to be superior to the MEL100 strategy with stem cell transplantation, a strategy with reduced intensity conditioning regimen. This result opens the debate on the role of stem cell transplantation in elderly patients in the era of novel agents. In patients over the age of 65 years $(n=136)$, the intensive Total Therapy program from the University of Arkansas achieved a median overall survival of 60 months with a $13 \%$ treatmentrelated mortality rate. ${ }^{26}$ In patients between 65 and 75 years of age, the oral MPT from the IFM achieved a median overall survival of 52 months with no toxic deaths.

\section{Toxicity}

Thalidomide induces a certain amount of hematopoietic and nonhematopoietic side effects, and MPT was associated with a significantly increased risk of complications in phase III trials comparing MP with MPT (Table 2). ${ }^{18,19}$ Certain complications are short-term side effects which are frequent, reversible, and manageable with appropriate dose reduction such as skin rash, sedation, constipation, sinus bradycardia, hypotension, and fatigue. Although teratogenicity should remain in everybody's mind as contraindicating thalidomide administration in pregnant women, it is not an issue in elderly women. Special recommendations were proposed for men and women of childbearing potential. ${ }^{27}$ Others are time- and dose-dependent. ${ }^{28}$ However, much has been learned in the past 8 years about the management of thalidomiderelated toxicities that are no longer a limitation in myeloma therapeutics for the majority of patients. ${ }^{29}$

In either studies from the IFM, a higher incidence of grade 3/4 neutropenia was noted but without an increase in severe infections. ${ }^{19,21}$ In contrast, no difference between MP and MPT was observed in the GIMEMA study for neutropenia, but severe infections were more frequent. ${ }^{18}$

Deep vein thrombosis (DVT) is a major adverse effect of thalidomide therapy, breakdown peripheral deep venous thrombosis and pulmonary embolism, the latter being of greater risk of morbidity. Its pathogenic mechanism has not been clearly established. When associated to dexamethasone or chemotherapy, an increased 
Table 2 Grade 3 and greater hematologic side effects, and incidence of deep venous thromboembolism and peripheral neuropathy of thalidomide in MM

\begin{tabular}{llllllll}
\hline Trial & Trial & $\mathbf{N}$ & $\begin{array}{l}\text { Thrombocytopenia } \\
(\%)\end{array}$ & $\begin{array}{l}\text { Neutropenia } \\
(\%)\end{array}$ & $\begin{array}{l}\text { Infection } \\
(\%)\end{array}$ & $\begin{array}{l}\text { DVT } \\
(\%)\end{array}$ & $\begin{array}{l}\text { Peripheral neuropathy } \\
(\%)\end{array}$ \\
\hline GIMEMA $^{18}$ & MPT & 129 & 3 & 16 & 10 & 12 & 8 \\
IFM 99-06' & MPT & 125 & 14 & 48 & 13 & 12 & 6 \\
IFM 0I-0I" $^{* 1}$ & MPT & 113 & ND & 0 & ND & 7 & 2 \\
\hline
\end{tabular}

Notes: "Grades 2 to 4; ' 'Leukopenia.

Abbreviations: $\mathrm{N}$, number of patients; ND, not determined.

risk is described. The outcome of DVT when treated only by thalidomide is estimated at 5\% and increases up to $15 \%$ when thalidomide is associated to either anthracycline or dexamethasone, in absence of any prophylactic anticoagulation. ${ }^{30-32}$ DVT have mainly been reported in the first 6 months of treatment with a large majority in the first 3 months when the burden of the tumor is the highest. ${ }^{33}$ Following such high incidence of DVT in the initial phases of studies, prophylactic anticoagulation was added. Clear thromboprophylaxis guidelines have been established for patients receiving a monotherapy by thalidomide or a combination including this drug when suffering of MM. ${ }^{25}$ Aspirin, low molecular weight heparin (LMWH), or oral warfarin should be initiated in all patients starting therapy with thalidomide plus dexamethasone and/or chemotherapy.

Peripheral neuropathy is the most common cause of thalidomide discontinuation or dose reduction and effects $15 \%$ to $20 \%$ of the patients. Moreover elderly patients suffer more frequently of this adverse side. Clinic manifestations are initially sensory symptoms but can evolve towards motor symptoms and autonomic dysfunction. Neuropathy is closely related to duration of treatment and cumulative dose. ${ }^{34-36}$ The probability of recovery increases if thalidomide is quickly discontinued. Otherwise neuropathic symptoms may progress and become irreversible. Practical rules were suggested recently by Palumbo in a review on thalidomide: no dose modification if neuropathy is grade I, $50 \%$ reduction dose if neuropathy is grade II, and interruption thalidomide if neuropathy is grade III or above. ${ }^{24}$

\section{MP + bortezomib (Velcade, MPV)}

The introduction of proteasome inhibition with bortezomib has expanded treatment options in MM and has significantly improved outcomes for patients with relapsed/refractory MM. ${ }^{37,38}$ The drug was also shown to be synergistic in vitro with a wide range of cytotoxic agents, including melphalan. ${ }^{39}$ In addition, the combination of bortezomib and melphalan was effective in a phase I/II trial. ${ }^{40}$ Based on these promising results, bortezomib was incorporated into the MP regimen (MPV) for the treatment of elderly untreated MM patients by the spanish group PETHEMA (Programa para el Tratamiento de Hemopatias Malignas). ${ }^{41}$ In total, 60 patients were enrolled in this phase I/II study, of whom 53 completed at least one cycle of treatment. The median age of patients was 75 years (range $65-85$ years); $47 \%$ of patients were older than 75 years and $17 \%$ were aged over 80 . Patients received four 6-week induction cycles (bortezomib 1.0 or $1.3 \mathrm{mg} / \mathrm{m}^{2}$ on days 1,4 , $8,11,22,25,29,32$ ) followed by five 5 -week maintenance cycles (bortezomib 1.0 or $1.3 \mathrm{mg} / \mathrm{m}^{2}$ on days $1,8,15,22$ ) in combination with oral melphalan $9 \mathrm{mg} / \mathrm{m}^{2}$ and prednisone $60 \mathrm{mg} / \mathrm{m}^{2} /$ day. The overall response rate was $89 \%$, including $32 \%$ of patients with immunofixation-negative CR and an additional $11 \%$ with near-CR. The response rate after 1 cycle of MPV was higher than in historical controls after 6 cycles of MP ( 70 versus $42 \%$ ). After a median follow-up of 26 months, median time to progression was 27.2 months, median overall survival had not been reached and projected 3 -year overall survival was $85 \%{ }^{42}$ Time-to-event data in the MPV group compared favourably with MP historical controls: time to progression, event-free survival and overall survival (at 26 months: 27.2 versus 20 months, 23 versus 16 months, and median not reached versus 26.9 months, respectively). MPV was generally well tolerated and the majority of adverse events occurred during the first 2 cycles of treatment. The most common grade- $3 / 4$ adverse events were thrombocytopenia and neutropenia $(40 \%-50 \%)$ but the frequency of infections was low (16\%). Peripheral neuropathy and diarrhea were noted in $17 \%$ and $16 \%$ of patients, respectively.

These promising results formed the basis from the international VISTA (Velcade as Initial Standard Therapy) trial (682 patients; the median age was 71 years with $30 \%$ of patients aged 75 years), which is so far the largest MP-based phase III study (Table 3). ${ }^{43}$ Patients received four 6-week induction cycles (bortezomib $1.3 \mathrm{mg} / \mathrm{m}^{2}$ on days $1,4,8,11$, $22,25,29,32)$ followed by five 6 -week maintenance cycles 
Table 3 Comparative response and survival in MPV compared with MP

\begin{tabular}{lllllll}
\hline Trial & Regimen & $\mathbf{N}$ & CR (\%) & PPR (\%) & TTP (mo) & OS (mo) \\
\hline VISTA $^{43}$ & MPV & 344 & 30 & 82 & 24 & $82.6 \%(2 \mathrm{yr})$ \\
& MP & 338 & 5 & 50 & 16.6 & $69.5 \%(2 \mathrm{yr})$ \\
\hline
\end{tabular}

Abbreviations: $\mathrm{N}$, number of patients; $\mathrm{CR}$, complete response; PR, partial response; OS, overall survival;TTP, time to progression.

(bortezomib $1.3 \mathrm{mg} / \mathrm{m}^{2}$ on days $1,8,22,29$ ) in combination with oral melphalan $9 \mathrm{mg} / \mathrm{m}^{2}$ and prednisone $60 \mathrm{mg} / \mathrm{m}^{2} /$ day. The primary endpoint was time to progression. MPV was found significantly superior to MP for all efficacy endpoints: time to progression, progression-free survival, overall survival, time to next treatment, CR rate. An unprecedented $30 \% \mathrm{CR}$ rate was noted in patients receiving MPV. Time to progression was 24 months in the MPV group. After a median follow-up of 16.3 months, median survival was significantly superior in the MPV group. The survival advantage was demonstrated in patients younger and older than 75 years of age. Serious adverse events were noted in $46 \%$ and $36 \%$ of patients in MPV and MP, respectively. Most divergent grade 3/4 toxicities between MPV and MP were gastrointestinal $(20 \%$ in MPV versus $6 \%$ in MP), fatigue and asthenia (15\% versus $5 \%$ ), and peripheral neuropathy ( $14 \%$ versus $0 \%)$. Peripheral neuropathy resolved or improved in $75 \%$ of cases in a median of approximately 60 days. Thrombosis/embolism was very low and the same on both arms (1\%). Overall, these results clearly established MPV as a new standard of care for MM patients not eligible for ASCT.

\section{MP + lenalidomide $\left(\right.$ Revlimid $\left.^{\circledR}\right)$}

Another successfully applied strategy has been the development of thalidomide derivatives potentially more potent compared to the parent compound and have fewer side-effects. Lenalidomide (Revlimid) ${ }^{\circledR}$ is a novel immunomodulatory agent that has shown activity both preclinically and clinically, ${ }^{44}$ alone or in combination with dexamethasone. ${ }^{45-47}$ Results from a phase I/II dose-escalating study of MP with lenalidomide (MPR) have been recently reported..$^{48}$ Fifty-four patients were enrolled in the study. The median age of patients was 71 years (range 57-77 years). Patients received 9 cycles of lenalidomide (5-10 mg/daily, days $1-21)$ plus melphalan $(0.18-0.25 \mathrm{mg} / \mathrm{kg} /$ day, days 1-4) and prednisone $2 \mathrm{mg} / \mathrm{kg} /$ day every 4-6 weeks, followed by maintenance therapy with lenalidomide alone (10 mg/daily, days $1-21$, every month). The maximum tolerated dose was lenalidomide $10 \mathrm{mg}$ /daily for 21 days and melphalan $0.18 \mathrm{mg} / \mathrm{kg}$ for 4 days every $4-6$ weeks. At this dose level, the overall response rate was $81 \%$ including
$48 \%$ of patients with at least a very good partial remission (VGPR; $>90 \%$ response rate) and $24 \%$ of patients with immunofixation-negative CR. The 1-year event-free survival and overall survival rates were $92 \%$ and $100 \%$, respectively. The most common grade $3 / 4$ adverse events were neutropenia and thrombocytopenia (52\% and $24 \%$, respectively at the maximum tolerated dose), febrile neutropenia (9\%), vasculitis (9\%), and thrombosis/embolism (5\%). More than $40 \%$ of patients required growth factor support. Overall, MPR was considered a promising first-line treatment for elderly patients with an acceptable and manageable toxicity. These results formed the basis for the ongoing European Myeloma Network phase III study comparing MP with MPR, with or without lenalidomide maintenance.

\section{Dexamethasone-based combinations}

Glucocorticoids have significant activity in MM, in relapsed/refractory disease and in newly diagnosed disease. In a study of 112 patients with untreated MM, the response rate with dexamethasone alone was $43 \%{ }^{14}$ In the 1990s, dexamethasone was considered a simple, effective, and safe primary treatment for a large fraction of patients, mainly in North America. In the course of time, high-dose dexamethasone was recognized as a major source of concern and disability for patients, especially in the elderly. An important contribution in that field was the IFM study IFM 95 for newly diagnosed myeloma patients aged $65-75$ years. In this study, 488 patients were randomly allocated to receive MP, melphalan with dexamethasone (M-Dex), dexamethasone alone, or dexamethasone with interferon alpha (Dex-IFN) (12). Patients receiving melphalan experienced a longer progression-free survival than those receiving dexamethasone-based regimens, but without any survival benefit. In addition, all high-dose dexamethasone-based regimens were associated with a greater risk of severe toxicities, including severe pyogenic infections in the M-Dex arm, and gastrointestinal, diabetic and psychiatric complications in all dexamethasone-based regimens. When combining all severe nonhematological complications, the incidence was significantly lower in 
the MP group (16\%) than in the dexamethasone-containing groups (28\%). Other study has demonstrated the higher incidence of adverse events in the MD arm. ${ }^{15}$

In a recent study from ECOG (Eastern Cooperative Oncology Group) comparing thalidomide plus dexamethasone (Thal/Dex) with dexamethasone alone in newly diagnosed patients, and even though the treatment duration was short and the study population included some young patients (median age 65 years, range 38-82), grade 3 or higher nonhematological toxicities were seen with $43 \%$ of patients with dexamethasone alone, and grade 3 or higher hyperglycemia, fatigue, insomnia, and muscle weakness were noted in $15 \%, 10 \%, 5 \%$, and $9 \%$ of patients, respectively. ${ }^{49}$ Toxicity of any type grade 4 or 5 was observed in $18 \%$ of patients including $4 \%$ treatment-related deaths. In addition, many other patients suffered from grade I/II dexamethasone-related toxicities which were also a source of disability. The toxicity of the Thal/Dex combination was higher than that observed with dexamethasone alone. Grade 3 or higher nonhematological toxicities were seen with $67 \%$ of patients within four cycles. Grade 4 or higher nonhematologic toxicities were seen with $35 \%$ of patients and treatment-related deaths in $5 \%$.

A confirmation of this significant toxicity was recently provided by the Central European Myeloma Study Group phase III study in elderly patients (289 patients; median age: 72 years) comparing Thal/Dex with MP. The study reported higher complete and near CR rates (30\% vs $14 \%$ ) for patients receiving Thal/Dex. ${ }^{50}$ Progression-free survival was similar in both groups (median 20.7 and 16.7 months for MP and Thal/Dex, respectively) but significantly shorter overall survival was observed in the Thal/Dex group (median 49.4 and 41.5 months for MP and Thal/Dex, respectively). The population was very elderly; especially in the Thal/Dex group with $60 \%$ of patients between the ages 70 and 79 and $10 \% \geq 80$ years. Patients received a high-dose dexamethasone regimen and thalidomide dosing was, up to $400 \mathrm{mg}$ /daily. Thus, the very elderly patient population and the higher doses of thalidomide and dexamethasone used likely contributed to a higher mortality rate in Thal/Dex-treated patients during the first year of study, especially in patients with a poorer performance status. When taken together with the MPT results from the Nordic Myeloma Study Group trial, these findings suggest that treatment with new drug combinations should be very carefully monitored in elderly patients, especially those over 75 years of age. Anthracyclins or alkylating agents have also been incorporated into the Thal/Dex regimen. ${ }^{51}$
The cyclophosphamide - thalidomide - dexamethasone (CTD) combination has proved to be effective in relapsed patients. ${ }^{52}$ These promising results prompted the Medical Research Council to proceed with a large phase III randomized study (MRC IX) comparing, in patients ineligible for transplantation, MP to CTD with an attenuated dexamethasone dose. Results from that study are eagerly anticipated.

Overall, when considering all these Thal/Dex experiences in terms of both efficacy and toxicity, there is evidence that this combination is inferior to MPT and may not be optimal for elderly patients.

Along with the frequent and serious dexamethasone side-effects, there were also data suggesting that high-doses of dexamethasone were possibly not necessary in combination with novel agents, such as thalidomide or lenalidomide..$^{53,54}$ The ECOG group proceeded recently with a study (ECOG E4A03) comparing lenalidomide plus high-dose dexamethasone (40 mg daily, days 1-4, 9-12, 17-20) with lenalidomide plus low-dose dexamethasone (40 mg daily, days 1, 8, 15, 22). ${ }^{55} 445$ patients, median age 66 years (up to 88 years), were treated, including 233 over the age of 65 years. The significant toxicity of the high-dose dexamethasone regimen was fully confirmed but the good news was the modest toxicity of the low-dose dexamethasone regimen. Infection/pneumonia, fatigue, hyperglycemia, deep venous thrombosis, and cardiac ischemia were significantly less frequent with the low-dose dexamethasone schedule. Overall, nonhematologic toxicity of any type grade $\geq 3$ or higher was found in $52 \%$ of patients receiving lenalidomide plus high-dose dexamethasone compared to $34 \%$ of patients receiving lenalidomide plus low-dose dexamethasone. Early deaths were also significantly less frequent in the low-dose dexamethasone arm; $1.4 \%$, versus $4.5 \%$. The two-year survival was superior in the group of patients receiving the low-dose dexamethasone regimen ( $87 \%$ versus $75 \%$ ), including in the subgroup of patients aged over 65 years ( $82 \%$ versus $67 \%$ ). Of note, the excess mortality in the high-dose dexamethasone arm was due to both disease progressive as well as increased toxicity. Overall, and even though the study was not designed to test efficacy of longterm lenalidomide plus dexamethasone (median durations on treatment were only 4 months in the high-dose dexamethasone arm and 6 months in the low-dose dexamethasone arm), lenalidomide and low-dose dexamethasone was found highly active in newly diagnosed elderly patients. There is no doubt that these results will be of major importance in the future and will influence the fate of all dexamethasone-based combinations. 


\section{Maintenance therapy in MM (Table 4)}

In the pre-thalidomide era, maintenance therapy ${ }^{56}$ with alkylating agents had failed to demonstrate any benefit ${ }^{57,58}$ as well as interferon which showed a modest increase in progression free survival and a minimal benefit in overall survival. ${ }^{59,60}$ Corticosteroid maintenance was found to prolong the duration of response but the effect on survival was controversial. ${ }^{61,62}$ Four randomized phase III trials evaluated the benefit of thalidomide maintenance after ASCT, ${ }^{63-66}$ and found thalidomide maintenance an effective approach following autologous stem cell transplantation in young patients (Table 4). Two ongoing studies are furthermore evaluating maintenance by thalidomide, the SouthWest Oncology Group, SWOG 0204 study ${ }^{67}$ and the HOVON-50/GMM-HD3 large trial, ${ }^{68}$ with only preliminary data available. Unfortunately, no large randomized study has looked so far specifically at older patients. In the near future, results from the MRC IX trial, which has included maintenance study comparing thalidomide versus observation-only, will be available. Several trials are also ongoing with lenalidomide maintenance.

\section{Supportive care therapy}

In symptomatic multiple myeloma, especially in elderly patients, most commonly experienced complications are asthenia, anorexia, and severe bone pain. Focal or diffuse bone involvement represents a common and disabling event in multiple myeloma. At diagnosis, bone pain in the skeleton, and particularly in the lumbar spine, represents the predominant symptom, $70 \%$ of patients, and is also a common indicator of relapse or progressive disease. In addition bone involvement contributes, along with immobility, dehydration and impaired renal calcium excretion, to the development of hypercalcemia. Anemia occurs in $30 \%$ to $62 \%$ of patients with multiple myeloma, particularly in those with advanced disease or delayed diagnosis, where it also has prognostic relevance. ${ }^{69}$
Infections are frequent complications of multiple myeloma and the most common causes of death due to suppression of production of polyclonal immunoglobulins, of decreased T-cell function and neutropenia. Such complications are a major cause of morbidity and mortality in myeloma patients, especially in elderly patients. Supportive care is therefore of critical importance in the treatment of elderly myeloma, in parallel with the specific treatment of myeloma.

\section{Skeletal-related complications}

Bone destruction in multiple myeloma (pathologic fractures, spinal cord compression, hypercalcemia) is a major cause of morbidity and significantly decrease quality of life, cause severe pain from bone lesions and neurological impairments. Although microfractures and pathologic fractures are common clinical manifestations in patients with multiple myeloma, they are rather infrequent. Pathologic fractures are usually site skeletal-related, with the majority involving vertebras $(55 \%$ to $70 \%$ ), lumbar and thoracic predominantly. ${ }^{4}$ Although bone destruction in elderly myeloma is not different from younger patients, the diagnosis of bone lesions related to myeloma might be difficult in elderly patients in the context of osteoporosis. ${ }^{6}$

The bone lesions-related pain described by patients with myeloma required specific treatment with effective analgesia. An effective analgesia is recommended using the WHO pain ladder to scale pain description from patients. Sufficient dosing and adequate scheduling of pain treatment are essential in order to ascertain sufficient and continuous pain control. Radiotherapy is also indicated for painful lesions: most patients significantly achieve pain relief with local radiotherapy at a dose of 30 grays in 10 to 15 fractions. Other indications include the treatment of impending or actual pathologic fractures, spinal cord compression, tumor causing local neurological problems, and large soft tissue plasma cell tumors. Radiotherapy has also been shown to prevent the development of new vertebral fractures. ${ }^{70}$

Table 4 Maintenance treatment with thalidomide

\begin{tabular}{|c|c|c|c|c|c|c|}
\hline Authors & N. of patients & $\begin{array}{l}\text { Duration } \\
\text { of treatment }\end{array}$ & $\begin{array}{l}\text { N. of } \\
\text { ASCT }\end{array}$ & $\begin{array}{l}\text { CR rate } \\
(\%)\end{array}$ & $\begin{array}{l}\text { PFS } \\
\text { (\%) }\end{array}$ & $\begin{array}{l}\text { OS } \\
(\%)\end{array}$ \\
\hline Barlogie et $\left.a\right|^{63}$ & 668 & $\begin{array}{l}\text { Onset until } \\
\text { relapse or } \\
\text { toxicity }\end{array}$ & double & 65 vs 43 & 56 vs 44 (5-year) & 65 vs 65 (5-year) \\
\hline IFM 99-0264 & 597 & $\begin{array}{l}\text { Until relapse } \\
\text { or toxicity }\end{array}$ & double & 67 vs 57 & 52 vs 36 or $37(3$-year) & 87 vs 74 or 77 (4-year) \\
\hline Australian Study ${ }^{65}$ & 243 & 12 months & single & 24 vs 15 & 63 vs 36 (2-year) & 51 vs 80 (2-year) \\
\hline Tunisian Study ${ }^{66}$ & 140 & 6 months & single & 67 vs 51 & 85 vs 57 & 85 vs 65 \\
\hline
\end{tabular}

Abbreviations: N., number; CR, complete response; PFS, progression-free survival; OS, overall survival;VS, versus. 
Surgical intervention may be required in patients with an impending or actual fracture or a spinal compression. ${ }^{71}$ Most patients also require radiotherapy in conjunction with surgery. Recent development of minimally invasive surgical procedures, such as vertebroplasty and kyphoplasty, allows myeloma patients with vertebral compression fractures to have immediate improvement in quality of life and shorter inpatient duration. ${ }^{72}$

Novel therapeutic approach for bone disease in multiple myeloma is represented by bisphosphonates which are derived from pyrophosphates by substitution of an oxygen atom with a carbon atom which confers a resistance to the activity of phosphatases in vivo, thus allowing prolonged inhibition of bone resorption. ${ }^{73,74}$ Bisphosphonates inhibit the recruitment of osteoclasts from their precursor cells and suppress their subsequent cellular proliferation and differentiation. They also bind to bone surfaces, thus protecting them from destruction, inhibit production of IL6, the most important growth hormone for myeloma cells, and stimulate apoptosis of osteoclasts and myeloma cells. The efficacy of the bisphosphonates clodronate, pamidronate, and zoledronate in preventing bone lesions has been investigated in several randomized trials: bisphosphonates decrease episodes of hypercalcemia, height loss, vertebral fractures, the development of new lytic lesions and pain. ${ }^{75,76}$ The addition of high potency intravenous bisphosphonates to myeloma therapy has provided major symptomatic benefits to patients with multiple myeloma. ${ }^{77}$ They also decrease the need for palliative radiation and recently presented data suggested that they may prolong time to disease progression. ${ }^{78}$

Osteonecrosis of the jaw (ONJ) is a major oral complication in patients with multiple myeloma secondary to intravenous bisphosphonates use $\mathrm{e}^{79-81}$ Intravenous bisphosphonates that contain an amino terminal group (pamidronate) or a nitrogen-containing side group (zoledronic acid) seem to present the highest risk for development of this phenomenon. Bisphosphonate-induced ONJ has been documented in both the maxilla and mandible, although there is a higher rate of incidence reported in the mandible. The median time to onset of ONJ among patients receiving zoledronic acid was 18 months and 3 to 6 years for patients receiving pamidronate. It is recommended that patients with multiple myeloma, who are being considered for IV bisphosphonates therapy, undergo a dental examination that includes clinical and radiographic evaluations. Any tooth or periodontal diseases should be appropriately controlled before initiation of IV bisphosphonates therapy. Dental extractions and other procedures that expose or manipulate bone should be avoided in these patients.
Due to the increasing risk of ONJ with duration of therapy, bisphosphonates should be discontinued after a maximum of 2 years of therapy in patients who have achieved CR and/or plateau phase. For patients whose disease is active, who have not achieved response, or who have a threatening bone disease beyond 2 years, therapy can be use to a dose every 3 months. ${ }^{82,83}$

\section{Anemia}

In general, anemia of multiple myeloma is normochomic, normo-macrocytic and especially at the disease onset, is often moderate and well-tolerated. In about $5 \%$ of the patients anemia may be the sole sign of the disease. The pathogenesis of anemia in multiple myeloma is multifactorial and causes vary with the phase of the disease. Plasma cell bone marrow infiltration represents the most important cause at diagnosis. However, other causes are hemodilution due to hypervolemia, reduced survival of erythrocytes, poor utilization of circulating iron, and renal failure. In addition, myeloma therapy, either chemotherapy or radiotherapy, may induce or worsen anemia. The role of a blunted production of endogenous erythropoietin (EPO) in anemia of MM patients has been demonstrated. ${ }^{69}$ In particular, reduced synthesis of EPO has been documented not only in MM subjects with renal failure (25\%), but also in patients with normal renal function. Cytokines such as IL-1 $\beta$ and tumor necrosis factor, produced by normal and neoplastic cells in MM, could inhibit the renal production of EPO while the role of the IL-6 is not clear in this context.

Several clinical trials have investigated the effects of recombinant EPO(r-EPO) in myeloma patients suffering from anemia. ${ }^{69}$ These studies indicate that r-EPO is an effective and safe drug for treating anemia of multiple myeloma. Quality of life in responding patients significantly improves with hemoglobin increment and transfusion independency. A functional or relative iron deficiency might occur in some patients during r-EPO treatment, and iron supplementation might be required to respond to $\mathrm{r}$-EPO therapy. ${ }^{84,85}$ Thromboembolic complications are the most important adverse event of EPO treatment. The risk is higher in patients with multiple myeloma treated with thalidomide or lenalidomide. ${ }^{86}$ Prophylactic anticoagulation with low-molecular-weight heparin or aspirin is recommended in these treatments. ${ }^{87}$

\section{Infections}

In early-stage myeloma, the most common infections are bronchitis and pneumonia, predominantly caused by 
Haemophilus influenzae and Streptococcus pneumoniae. In patients with advanced myeloma and during the neutropenic phases of chemotherapy, S. aureus and Gram-negative bacteria are more common. Treatment is based on antibiotics. ${ }^{6}$ Vaccination against $H$. influenzae and $S$. pneumoniae is recommended, with $61 \%$ of patients producing protective antibodies against pneumococci and $75 \%$ against $H$. influenzae. A randomized trial showed that prophylaxis of infection with monthly immunoglobulin infusions $(0.4 \mathrm{~g} / \mathrm{kg})$ for a period of one year significantly reduced the frequency and severity of infections with patients who responded poorly to pneumococcal immunization.

\section{Conclusion}

Large randomized phase III studies have now established MPT and MPV as new standards of care for a large proportion of elderly MM patients ineligible for ASCT. MPR may also provide a survival advantage over MP. This potentially may lead to the availability of three therapeutic options for the treatment of newly diagnosed elderly patients with MM. These options could lead to more personalized treatment approaches, based on patient comorbidities, as the three novel agents have somewhat different toxicity profiles. Physicians should also keep in mind that an adequate symptomatic treatment remains essential as well as a very careful treatment monitoring. The dexamethasone history, and also some recent experiences with new drugs have shown that the highest doses of drugs are not always optimal in elderly patients. All efforts should be made to avoid excessive toxicity, particularly in patients with initial bad performance status, and early phases of treatment are critical. Outpatient administration of drugs and patient quality of life should also be favored.

\section{Disclosure}

The authors report no conflicts of interest in this work.

\section{References}

1. Jemal A, Murray T, Ward E, et al. Cancer statistics. CA Cancer J Clin. 2005;55:10-30.

2. Kyle RA, Rajkumar SV. Multiple myeloma. $N$ Engl J Med. 2004;351:1860-73.

3. Jawed I, Lee CM, Tward JD, et al. Survival outcomes for multiple myeloma over three decades: A Surveillance, Epidemiology, and End Results (SEER) analysis. (\#8019). J Clin Oncol, 2007. ASCO Annual Meeting Proceedings. No. 18S (June 20 Supplement). 2007;25 Part I.

4. Anagnostopoulos A, Gika D, Symeonidis A, et al. Multiple myeloma in elderly patients: prognostic factors and outcome. Eur J Haematol. 2005;75:370-5.

5. Ludwig H, Durie BG, Bolejack V, et al. Myeloma in patients younger than age 50 years presents with more favorable features and shows better survival: an analysis of 10549 patients from the International Myeloma Working Group. Blood. 2008;111:4039-47.
6. Ludwig H, Zojer N. Supportive care in multiple myeloma. Best Pract Res Clin Haematol. 2007;20:817-35.

7. Attal M, Harousseau JL, Stoppa AM, et al. A prospective, randomized trial of autologous bone marrow transplantation and chemotherapy in multiple myeloma. Intergroupe Francais du Myelome. $N$ Engl J Med. 1996;335:91-7.

8. Badros A, Barlogie B, Siegel E, et al. Autologous stem cell transplantation in elderly multiple myeloma patients over the age of 70 years. Br J Haematol. 2001;114:600-7.

9. Jantunen E, Itala M, Juvonen E, et al. Autologous stem cell transplantation in elderly ( $>60$ years) patients with non-Hodgkin's lymphoma: a nation-wide analysis. Bone Marrow Transplant. 2006;37:367-72.

10. Alexanian R, Haut A, Khan AU, et al. Treatment for multiple myeloma. Combination chemotherapy with different melphalan dose regimens. JAMA. 1969;208:1680-5.

11. Bataille R, Harousseau JL. Multiple myeloma. $N$ Engl J Med. 1997;336:1657-64.

12. Facon T, Mary JY, Pegourie B, et al. Dexamethasone-based regimens versus melphalan-prednisone for elderly multiple myeloma patients ineligible for high-dose therapy. Blood. 2006;107:1292-8.

13. Group MTC. Combination chemotherapy versus melphalan plus prednisone as treatment for multiple myeloma: an overview of 6,633 patients from 27 randomized trials. Myeloma Trialists' Collaborative Group. J Clin Oncol. 1998;16:3832-42.

14. Alexanian R, Dimopoulos MA, Delasalle K, et al. Primary dexamethasone treatment of multiple myeloma. Blood. 1992;80:887-90.

15. Hernandez JM, Garcia-Sanz R, Golvano E, et al. Randomized comparison of dexamethasone combined with melphalan versus melphalan with prednisone in the treatment of elderly patients with multiple myeloma. Br J Haematol. 2004;127:159-64.

16. Singhal S, Mehta J, Desikan R, et al. Antitumor activity of thalidomide in refractory multiple myeloma. $N$ Engl J Med. 1999;341:1565-71.

17. Palumbo A, Bertola A, Musto P, et al. Oral melphalan, prednisone, and thalidomide for newly diagnosed patients with myeloma. Cancer. 2005; 104:1428-33.

18. Palumbo A, Bringhen S, Caravita T, et al. Oral melphalan and prednisone chemotherapy plus thalidomide compared with melphalan and prednisone alone in elderly patients with multiple myeloma: randomised controlled trial. Lancet. 2006;367:825-31.

19. Facon T, Mary JY, Hulin C, et al. Melphalan and prednisone plus thalidomide versus melphalan and prednisone alone or reducedintensity autologous stem cell transplantation in elderly patients with multiple myeloma (IFM 99-06): a randomised trial. Lancet. 2007;370:1209-18.

20. Palumbo A, Bringhen S, Liberati AM, et al. Oral melphalan, prednisone, and thalidomide in elderly patients with multiple myeloma: updated results of a randomized, controlled trial. Blood. 2008; DOI 10.1182/ blood-2008-04-149427.

21. Hulin C, Facon T, Rodon P, et al. Melphalan-prednisone-thalidomide (MP-T) demonstrates a significant survival advantage in elderly patients $\geq 75$ years with multiple myeloma compared with melphalanprednisone (MP) in a randomized, double-blind, placebo-controlled trial, IFM 01/01. Blood. 2007;110.

22. Waage A, Gimsing P, Juliusson G, et al. Melphalan-prednisone-thalidomide to newly diagnosed patients with multiple myeloma: a placebo controlled randomised phase 3 trial. Blood. 2008;110.

23. Wijermans P, Schaafsma P, Van Norden Y, et al. Melphalan Prednisone versus Melphalan Prednisone Thalidomide in induction therapy for Multiple Myeloma in Elderly patients: First interim results of the Dutch cooperative group HOVON. (\#0440). Haematologica. 2008;93(s1):178.

24. Palumbo A, Facon T, Sonneveld P, et al. Thalidomide for treatment of multiple myeloma: 10 years later. Blood. 2008;111:3968-77.

25. Palumbo A, Rajkumar SV, Dimopoulos MA, et al. Prevention of thalidomide- and lenalidomide-associated thrombosis in myeloma. Leukemia. 2008;22:414-23. 
26. Pineda-Roman M, Barlogie B, Anaissie E, et al. High-dose melphalan-based autotransplants for multiple myeloma: the Arkansas experience since 1989 in 3077 patients. Cancer. 2008;112:1754-64.

27. Stephens TD, Bunde CJ, Fillmore BJ. Mechanism of action in thalidomide teratogenesis. Biochem Pharmacol. 2000;59:1489-99.

28. Matthews SJ, McCoy C. Thalidomide: a review of approved and investigational uses. Clin Ther. 2003;25:342-95.

29. Ghobrial IM, Dispenzieri A, Bundy KL, et al. Effect of thalidomide on stem cell collection and engraftment in patients with multiple myeloma. Bone Marrow Transplant. 2003;32:587-92.

30. Barlogie B, Desikan R, Eddlemon P, et al. Extended survival in advanced and refractory multiple myeloma after single-agent thalidomide: identification of prognostic factors in a phase 2 study of 169 patients. Blood. 2001;98:492-4.

31. Rajkumar SV, Hayman S, Gertz MA, et al. Combination therapy with thalidomide plus dexamethasone for newly diagnosed myeloma. J Clin Oncol. 2002;20:4319-23.

32. Zangari M, Siegel E, Barlogie B, et al. Thrombogenic activity of doxorubicin in myeloma patients receiving thalidomide: implications for therapy. Blood. 2002;100:1168-71.

33. Zangari M, Anaissie E, Barlogie B, et al. Increased risk of deep-vein thrombosis in patients with multiple myeloma receiving thalidomide and chemotherapy. Blood. 2001;98:1614-58.

34. Cavaletti G, Beronio A, Reni L, et al. Thalidomide sensory neurotoxicity: a clinical and neurophysiologic study. Neurology. 2004;62:2291-3.

35. Richardson P, Schlossman R, Jagannath S, et al. Thalidomide for patients with relapsed multiple myeloma after high-dose chemotherapy and stem cell transplantation: results of an open-label multicenter phase 2 study of efficacy, toxicity, and biological activity. Mayo Clin Proc. 2004;79:875-82.

36. Mileshkin L, Stark R, Day B, et al. Development of neuropathy in patients with myeloma treated with thalidomide: patterns of occurrence and the role of electrophysiologic monitoring. J Clin Oncol. 2006;24:4507-14.

37. Richardson PG, Barlogie B, Berenson J, et al. A phase 2 study of bortezomib in relapsed, refractory myeloma. N Engl J Med. 2003;348:2609-17.

38. Richardson PG, Sonneveld P, Schuster MW, et al. Bortezomib or highdose dexamethasone for relapsed multiple myeloma. $N$ Engl J Med. 2005;352:2487-98.

39. Mitsiades N, Mitsiades CS, Richardson PG, et al. The proteasome inhibitor PS-341 potentiates sensitivity of multiple myeloma cells to conventional chemotherapeutic agents: therapeutic applications. Blood. 2003;101:2377-80.

40. Berenson JR, Yang HH, Sadler K, et al. Phase I/II trial assessing bortezomib and melphalan combination therapy for the treatment of patients with relapsed or refractory multiple myeloma. J Clin Oncol. 2006;24:937-44.

41. Mateos MV, Hernandez JM, Hernandez MT, et al. Bortezomib plus melphalan and prednisone in elderly untreated patients with multiple myeloma: results of a multicenter phase I/II study. Blood. 2006;108:2165-72.

42. Mateos MV, Hernandez JM, Hernandez MT, et al. Bortezomib plus melphalan and prednisone in elderly untreated patients with multiple myeloma: updated time-to-events results and prognostic factors for time to progression. Haematologica. 2008;93:560-5.

43. San Miguel J, Schlag R, Khuageva N, et al. MMY-3002: A Phase 3 study comparing bortezomib-melphalan-prednisone (VMP) with melphalan-prednisone (MP) in newly diagnosed multiple myeloma. Blood. 2007;110.

44. Richardson P, Anderson K. Immunomodulatory analogs of thalidomide: an emerging new therapy in myeloma. J Clin Oncol. 2004;22:3212-4.

45. Mitsiades N, Mitsiades CS, Poulaki V, et al. Apoptotic signaling induced by immunomodulatory thalidomide analogs in human multiple myeloma cells: therapeutic implications. Blood. 2002;99:4525-30.

46. Richardson PG, Schlossman RL, Weller E, et al. Immunomodulatory drug CC-5013 overcomes drug resistance and is well tolerated in patients with relapsed multiple myeloma. Blood. 2002;100:3063-7.
47. Rajkumar SV, Hayman S, Lacy MQ, et al. Combination therapy with CC-5013 (Lenalidomide, RevlimidTM) plus dexamethasone (Rev/ Dex) for newly diagnosed myeloma (MM). Blood. 2004;104:98a.

48. Palumbo A, Falco P, Falcone A, et al. Oral Revlimid ${ }^{\circledR}$ plus melphalan and prednisone (R-MP) for newly diagnosed multiple myeloma: results of a multicenter phase I/II study. Blood. 2007;108.

49. Rajkumar SV, Blood E, Vesole D, et al. Phase III clinical trial of thalidomide plus dexamethasone compared with dexamethasone alone in newly diagnosed multiple myeloma: a clinical trial coordinated by the Eastern Cooperative Oncology Group. J Clin Oncol. 2006;24:431-6.

50. Ludwig H, Tothova E, Hajek R, et al. Thalidomide-dexamethasone vs melphalan-prednisone as first line treatment and thalidomide-interferon vs interferon maintenance therapy in elderly patients with multiple myeloma. Blood. 2007;110.

51. Offidani M, Corvatta L, Marconi M, et al. Low-dose thalidomide with pegylated liposomal doxorubicin and high-dose dexamethasone for relapsed/refractory multiple myeloma: a prospective, multicenter, phase II study. Haematologica. 2006;91:133-6.

52. Kyriakou C, Thomson K, D'Sa S, et al. Low-dose thalidomide in combination with oral weekly cyclophosphamide and pulsed dexamethasone is a well tolerated and effective regimen in patients with relapsed and refractory multiple myeloma. Br J Haematol. 2005;129:763-70.

53. Palumbo A, Giaccone L, Bertola A, et al. Low-dose thalidomide plus dexamethasone is an effective salvage therapy for advanced myeloma. Haematologica. 2001;86:399-403.

54. Coleman M, Leonard J, Lyons L, et al. BLT-D (clarithromycin [Biaxin], low-dose thalidomide, and dexamethasone) for the treatment of myeloma and Waldenstrom's macroglobulinemia. Leuk Lymphoma. 2002;43:1777-82.

55. Rajkumar S, Jacobus S, Callander N, et al. A randomized trial of lenalidomide plus high-dose dexamethasone (RD) versus lenalidomide plus low-dose dexamethasone ( $\mathrm{Rd}$ ) in newly diagnosed multiple myeloma (E4A03): A trial coordinated by the Eastern Cooperative Oncology Group. Blood. 2007;110.

56. Harousseau JL. Maintenance treatment in multiple myeloma. Ann Oncol. 2008;19(Suppl 4):iv54-5.

57. Alexanian R, Balcerzak S, Bonnet JD, et al. Prognostic factors in multiple myeloma. Cancer. 1975;36:1192-201.

58. Belch A, Shelley W, Bergsagel D, et al. A randomized trial of maintenance versus no maintenance melphalan and prednisone in responding multiple myeloma patients. Br J Cancer. 1988;57:94-9.

59. Fritz E, Ludwig H. Interferon-alpha treatment in multiple myeloma: meta-analysis of 30 randomised trials among 3948 patients. Ann Oncol. 2000;11:1427-36.

60. Schaar CG, Kluin-Nelemans HC, Te Marvelde C, et al. Interferon-alpha as maintenance therapy in patients with multiple myeloma. Ann Oncol. 2005;16:634-9.

61. Berenson JR, Crowley JJ, Grogan TM, et al. Maintenance therapy with alternate-day prednisone improves survival in multiple myeloma patients. Blood. 2002;99:3163-8.

62. Shustik C, Belch A, Robinson S, et al. A randomised comparison of melphalan with prednisone or dexamethasone as induction therapy and dexamethasone or observation as maintenance therapy in multiple myeloma: NCIC CTG MY.7. Br J Haematol. 2007;136:203-11.

63. Barlogie B, Tricot G, Anaissie E, et al. Thalidomide and hematopoietic-cell transplantation for multiple myeloma. $N$ Engl J Med. 2006;354:1021-30.

64. Attal M, Harousseau JL, Leyvraz S, et al. Maintenance therapy with thalidomide improves survival in patients with multiple myeloma. Blood. 2006;108:3289-94.

65. Spencer A, Prince HM, Roberts A, et al. Thalidomide improves survival when used following ASCT. Haematologica. 2007;92(Suppl 2):S7b.5.

66. Abdelkefi A, Ladeb S, Torjman L, et al. Single autologous stem-cell transplantation followed by maintenance therapy with thalidomide is superior to double autologous transplantation in multiple myeloma: results of a multicenter randomized clinical trial. Blood. 2008;111:1805-10. 
67. Hussein M, Jakubowiak AJ, Bolejack V, et al. S0204: Melphalan (MEL)-based tandem autotransplants (TAT) for multiple myeloma (MM) with thalidomide/dexamethasone (TD) induction and thalidomide/ prednisone (TP) maintenance: A phase II trial of the Southwest Oncology Group. Blood. 2006;108.

68. Goldschmidt H, Sonneveld P, Cremer FW, et al. Joint HOVON-50/ GMMG-HD3 randomized trial on the effect of thalidomide as part of a high-dose therapy regimen and as maintenance treatment for newly diagnosed myeloma patients. Ann Hematol. 2003;82:654-9.

69. Ludwig H, Pohl G, Osterborg A. Anemia in multiple myeloma. Clin Adv Hematol Oncol. 2004;2:233-41.

70. Leigh BR, Kurtts TA, Mack CF, et al. Radiation therapy for the palliation of multiple myeloma. Int J Radiat Oncol Biol Phys. 1993;25:801-4.

71. Spinazze S, Caraceni A, Schrijvers D. Epidural spinal cord compression. Crit Rev Oncol Hematol. 2005;56:397-406.

72. Yeh HS, Berenson JR. Treatment for myeloma bone disease. Clin Cancer Res. 2006;12(20 Pt 2):6279s-84s.

73. Kimmel DB. Mechanism of action, pharmacokinetic and pharmacodynamic profile, and clinical applications of nitrogencontaining bisphosphonates. J Dent Res. 2007;86:1022-33.

74. Russell RG, Xia Z, Dunford JE, et al. Bisphosphonates: an update on mechanisms of action and how these relate to clinical efficacy. Ann N Y Acad Sci. 2007;1117:209-57.

75. Musto P, Falcone A, Sanpaolo G, et al. Pamidronate reduces skeletal events but does not improve progression-free survival in early-stage untreated myeloma: results of a randomized trial. Leuk Lymphoma. $2003 ; 44: 1545-8$.

76. Rosen LS, Gordon D, Kaminski M, et al. Long-term efficacy and safety of zoledronic acid compared with pamidronate disodium in the treatment of skeletal complications in patients with advanced multiple myeloma or breast carcinoma: a randomized, double-blind, multicenter, comparative trial. Cancer. 2003;98:1735-44.

77. Stoopler ET, Vogl DT, Stadtmauer EA. Medical management update multiple myeloma. Oral Surg Oral Med Oral Pathol Oral Radiol Endod. 2007;103:599-609.
78. Berenson JR, Lichtenstein A, Porter L, et al. Long-term pamidronate treatment of advanced multiple myeloma patients reduces skeletal events. Myeloma Aredia Study Group. J Clin Oncol. 1998;16:593-602.

79. Bamias A, Kastritis E, Bamia C, et al. Osteonecrosis of the jaw in cancer after treatment with bisphosphonates: incidence and risk factors. $J$ Clin Oncol. 2005;23:8580-7.

80. Durie BG, Katz M, Crowley J. Osteonecrosis of the jaw and bisphosphonates. $N$ Engl J Med. 2005;353:99-102; Discussion 199-202.

81. Dimopoulos MA, Kastritis E, Anagnostopoulos A, et al. Osteonecrosis of the jaw in patients with multiple myeloma treated with bisphosphonates: evidence of increased risk after treatment with zoledronic acid. Haematologica. 2006;91:968-71.

82. Lacy MQ, Dispenzieri A, Gertz MA, et al. Mayo clinic consensus statement for the use of bisphosphonates in multiple myeloma. Mayo Clin Proc. 2006;81:1047-53.

83. Kyle RA, Yee GC. Somerfield MR, et al. American Society of Clinical Oncology 2007 clinical practice guideline update on the role of bisphosphonates in multiple myeloma. J Clin Oncol. 2007;25:2464-72.

84. Osterborg A, Boogaerts MA, Cimino R, et al. Recombinant human erythropoietin in transfusion-dependent anemic patients with multiple myeloma and non-Hodgkin's lymphoma - a randomized multicenter study. The European Study Group of Erythropoietin (Epoetin Beta) Treatment in Multiple Myeloma and Non-Hodgkin's Lymphoma. Blood. 1996;87:2675-82.

85. Bokemeyer C, Aapro MS, Courdi A, et al. EORTC guidelines for the use of erythropoietic proteins in anaemic patients with cancer: 2006 update. Eur J Cancer. 2007;43:258-70.

86. Knight R, DeLap RJ, Zeldis JB. Lenalidomide and venous thrombosis in multiple myeloma. $N$ Engl J Med. 2006;354:2079-80.

87. Menon SP, Rajkumar SV, Lacy M, et al. Thromboembolic events with lenalidomide-based therapy for multiple myeloma. Cancer. $2008 ; 112: 1522-8$ 
